\title{
Deformation Aging in Low Carbon Steels
}

\author{
Sergey Mikhailov ${ }^{1, *}$, Valentina Sharapova ${ }^{1}$, Ekaterina Skobelina $^{1}$, and Leonid Molokov ${ }^{1}$ \\ ${ }^{1}$ Ural Federal University (UrFU), 620002 Ekaterinburg, 19 Mira st., Russia
}

\begin{abstract}
The results of mechanical spectroscopy are shown when studying the processes of strain age-hardening in mild steel with a ferritebainitic structure.
\end{abstract}

\section{Introduction}

The Snook peak $\left(50 \ldots 60^{\circ} \mathrm{C}\right)$ and the Snook-Köster peak $\left(200 \ldots 250^{\circ} \mathrm{C}\right)$ are used to assess the strain aging of low-carbon steels with ferrite in the structure. These peaks can be recorded by mechanical spectroscopy techniques [1] for temperature-dependent internal friction (TDI) $[2,3]$. These measurements provide information on the degradation of the mechanical properties of steel during their long-term operation [3].

Currently, a number of industries use low-carbon steels with a ferrite-pearlite structure (FP). The use of heat treatment with accelerated cooling from the austenitic region leads to the formation of a ferrite-bainitic (F-B) structure. This structure has higher strength properties [4]. The problem of analyzing the behavior of the noted peaks of temperaturedependent internal friction (TDI) in the case of replacing a ferrite-pearlite (F-P) structure with a ferrite-bainitic (F-B) one for the same steel is urgent.

\section{Materials and methods}

Samples 06G2FB with ferrite-pearlite (F-P) and ferrite-bainitic (F-B) was tested with a system for holding a straight torsion pendulum. The massive sample has a cylindrical 5-fold working part with a diameter of $6 \mathrm{~mm}$, at a resonance frequency of $36 \mathrm{~Hz}$, with the imposition of a constant magnetic field along the axis of the sample. Preparation and certification of the structure of the samples were carried out similarly to the samples of work [4].

Additionally, the sample with the F-B structure was tempered in the installation for 1 hour in the temperature range from 20 to $700^{\circ} \mathrm{C}$, with a sequential step of $100{ }^{\circ} \mathrm{C}$ increase. After each tempering, a cycle of measurements of the amplitude-dependent internal friction (ADI) was carried out according to the scheme ("excitation", "maturation", tempering at a higher temperature). "Excitation" included elastic deformation cyclic torsion at an amplitude level of $0.25 \%$ shear for 12 minutes. Subsequent "aging" was carried out for 7 days at room temperature. It should be noted that the amplitude level of $0.3 \%$ shear corresponds to the conventional yield stress in torsion. The test was carried out sequentially

\footnotetext{
* Corresponding author: msb.immt@gmail.com
} 
without changing the position of the sample in the measuring cell of the setup to minimize the error.

\section{Discussions}

Figure 1 shows the results of measurements of temperature-dependent internal friction obtained on a sample with a ferrite-pearlite structure. The sample was preheated during tempering at $135{ }^{\circ} \mathrm{C}$ in order to reduce the effect of mechanical turning during the manufacture of the working part of the sample. Measurements of the temperature dependences of the attenuation (inverse Q-factor - Q-1) and the shear modulus level (relative defect of the shear modulus - dG) were carried out without (1-Q-1 b / p, 1-dG b / p) magnetic field, as well as with superposition magnetic field (2-Q-1 in $\mathrm{m} / \mathrm{n}, 2-\mathrm{dG}$ in $\mathrm{m} /$ n). An increase in the vertical scale of the curve 2-Q-1 in a magnetic field (Fig.1b) makes it possible to clearly record two peaks for the P-P structure with a maximum at $100{ }^{\circ} \mathrm{C}$ and $300{ }^{\circ} \mathrm{C}$. Taking into account that an increase in the measurement frequency from $1 \mathrm{~Hz}$ to 36 $\mathrm{Hz}$ should contribute to the shift of relaxation peaks to higher temperatures, we believe that the identified peaks on the curve 2-Q-1 in a magnetic field (Fig. 1b) refer to the peak of Snook and Snook. Köster, respectively.

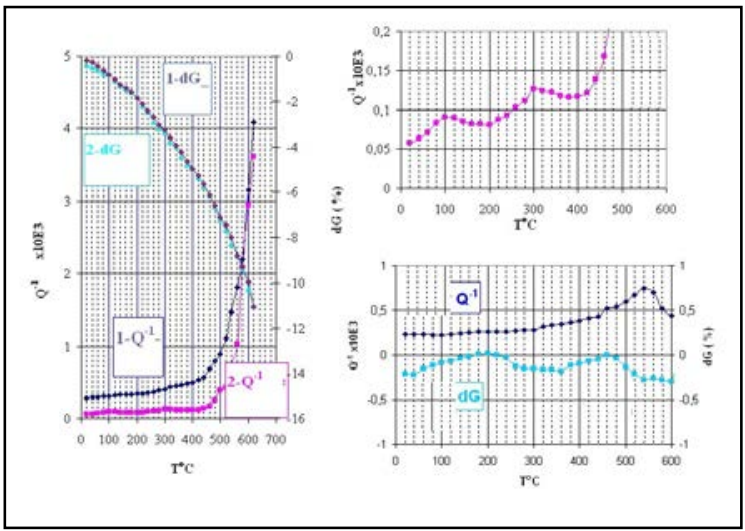

Fig. 1. Temperature-dependent internal friction of a $06 \mathrm{G} 2 \mathrm{FB}$ steel sample with a ferrite-pearlite structure after tempering at $135^{\circ} \mathrm{C}, 1$ hour.

In this case, the height of the Snook peak characterizes the content of dissolved interstitial atoms in ferrite, and the height of the Snook-Köster peak characterizes the interaction of interstitial atoms with dislocations in the sample. In addition, the peak in the temperature range of $250 \ldots 350{ }^{\circ} \mathrm{C}$ is due to the relaxation of microstresses in martensite during its heating during tempering in the above temperature range.

The difference between the curves shown in Fig. 1a reflects the magnetoelastic components ( $\mathrm{m} / \mathrm{y}$ Q-1, $\mathrm{m} / \mathrm{y} \mathrm{dG}$ ) associated with the mobility of the domain walls of the ferromagnetic state of the sample (Fig. 1c). 
The measurements showed that with the transition from the F-P structure to the F-B, cardinal changes occur in the results of the measurements obtained (Fig. 2). So, for the F-P structures, there are approximately equal peaks of both Snook and Snook-Koester (Fig. 1b). At the same time, an increase in the tempering temperature modifies the height and position of the Snook peak, increasing and shifting down (Fig. 2a_curve 2-Q-1 in $\mathrm{m} / \mathrm{n} \_135{ }^{\circ} \mathrm{C}$ ) relative to the delivery state (Fig. 2a_curve 2-Q-1 in m/n_20 ${ }^{\circ} \mathrm{C}$ ). With an increase in the tempering temperature to $240{ }^{\circ} \mathrm{C}$, the opposite process takes place, which is enhanced by plastic deformation by stretching of 3\% (Fig. 2a_Control) before tempering.

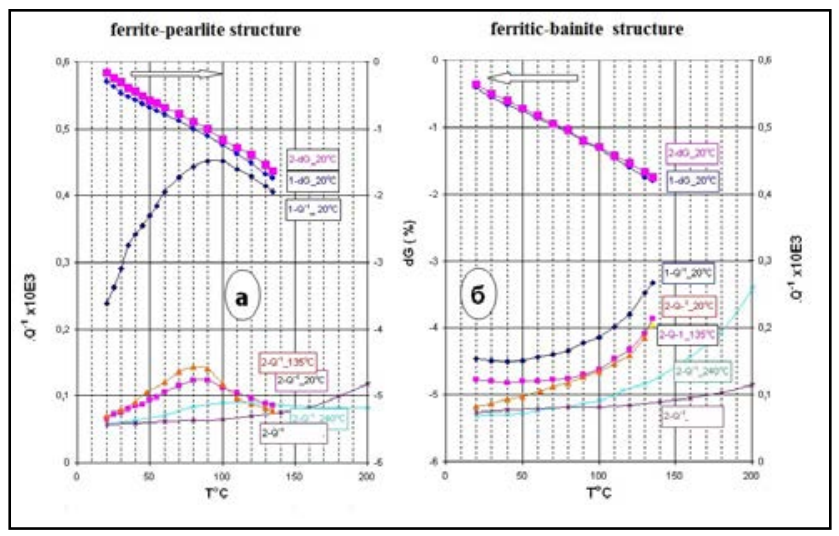

Fig. 2. Influence of the initial structure of the sample of low-carbon steel 06G2FB on the Snook peak after different tempering temperatures: $\mathrm{a}$ - ferritic-pearlite structure, $\mathrm{b}$ - ferritic-bainite structure.

The noted changes are standard manifestations of the effects of strain aging $[2,3]$.

With the transition to the F-B structure (Fig. 2b), the Snook peak is absent up to a tempering temperature of $700{ }^{\circ} \mathrm{C}$. A change in the overall level of the values of the TDI magnetoelastic components is observed synchronously. So, for the F-B structure, the values of this level are seven times lower in attenuation and about three times higher in terms of the module defect. Noteworthy is the existence of narrow temperature regions of an underestimated level of magnetoelastic damping in F-B structures at tempering temperatures corresponding to the initial stages of secondary hardening associated with alloying steel with vanadium and niobium.

In addition to this, a significant decrease in the level of magnetoelastic damping takes place in the P-P structure after tempering at $250^{\circ} \mathrm{C}$. It should be noted that this is the same sample and the appearance of the Snook peak in it is not associated with anomalies in the chemical composition, but is caused by the heat treatment regime, accompanied by significant decarburization of the ferrite structure and relaxation of internal microstresses.

\section{Conclusions}

The absence of the Snook peak in the ferrite component of the F-B structure of our sample is a somewhat unexpected fact, which contradicts the experiments of [3]. However, it should be taken into account that up to $40 \%$ of equilibrium ferrite was present in the structure of the sample in [3]. In our sample, this phase was significantly less. The increased carbon content in the ferrite of the sample with the F-B structure should contribute to the appearance of a high level of microdistortions. This is indicated by the "giant" 200-degree peak of Snook-Köster.

Carbide formation is accompanied by the release of carbon from the solid solution and relaxation of microstresses at elevated tempering temperatures. With cyclic excitation, the 
formation of the Snook peak is possible due to the uniform distribution of carbon atoms over octahedral pores.

Thus, the traditionally used experimental technique for studying strain aging by changing the parameters of the Snook peak with an increased carbon content in ferrite is ineffective.

\section{References}

1. I.S. Golovin, Internal friction and mechanical spectroscopy of metallic materials: textbook (Ed. House of MISIS, Moscow, 2012)

2. S.O. Suvorova, V.I. Sarrak, R.I. Entin, PhMM, 17, 1, 105-111 (1964)

3. G.A. Filippov, V.N. Zikeev, I.P. Shabalov, O.V. Livanova, A.R. Mishetyan, Probl. of ferr. met. and mat. sci., 1, 49-55 (2017)

4. M.A. Smirnov, I. Yu. Pyshmintsev, O.V. Varnak, A.N. Maltseva, Yu.N. Goichenberg, STEEL, 1, 39-45 (2016) 